\title{
Angina tonsillaris - Antibiotika ja oder nein?
}

\author{
Philippe Rafeiner \\ FMH Innere Medizin und Infektiologie, Innere Medizin und Diagnostik, Spital Zofingen
}

Gerber, Wäckerlin und Flury beschreiben trefflich anhand eines Fallbeispiels das schwergradige Krankheitsbild eines Lemierre-Syndroms [1]. Letzteres ist Ausdruck einer septischen Thrombophlebitis der V. jugularis und entsteht meist sekundär durch Fusibacterium necrophorum im Rahmen einer primären Tonsillo-Pharyngitis. Das Lemierre-Syndrom ist mit einer Inzidenz von 1 bzw. 14 Fällen/Mio. Erwachsenen bzw. Adoleszenten selten, aber vom Krankheitsausmass und der Letalität her bedeutend.

Anamnestisch besteht als nahezu pathognomonisches Merkmal eine zweigipflige Manifestation einer TonsilloPharyngitis (meist wenige Tage nach Regredienz oder Abklingen von Halsschmerzen Wiederauftreten von Beschwerden). Daneben kann auch der prolongierte Verlauf einer Angina Ausdruck eines beginnenden LemierreSyndroms sein.

Bekanntlich stellt die bakteriell bedingte Tonsillo-Pharyngitis statistisch gesehen im Vergleich zur viralen Genese eine Ausnahme dar (Häufigkeit in Abhängigkeit der Altersgruppe 5-30\%). Es stellt sich grundsätzlich die berechtigte Frage, ob bei bakteriell bedingter Angina tonsillaris durch vor- oder frühzeitige Gabe von Antibiotika Komplikationen vermieden werden können. Zur Beurteilung eines potenziellen Nutzens bedarf es einerseits der «Number Needed to Treat» (NNT), der «Number Treated to Harm» (NNH) und der Einschätzung der Gefährlichkeit der Komplikation.

Betreffend der Verhütung von Komplikationen einer durch Streptokokken Gruppe A (GAS) bedingten Tonsillitis herrscht zumindest in gewissen europäischen Ländern zunehmend der Konsens, dass die antibiotische Behandlung (gemessen an den oben genannten Parametern) insgesamt keinen nennenswerten Benefit zu haben scheint (keine gesicherte Verhütung eines Peritonsillarabszesses, eine Komplikation, die, rechtzeitig erkannt, insgesamt eine gute Prognose aufweist; keine sichere Verminderung des ohnehin bei uns sehr seltenen rheumatischen Fiebers) [2]. 2019 hat auch eine Schweizer Arbeitsgruppe diese Sichtweise publiziert [3].

Gerber, Wäckerlin und Flury propagieren gemäss Centor et al. [4] einen Benefit einer grosszügigen antibiotischen Behandlung im Fall von mindestens zwei positiven Centor-Kriterien in der Altersgruppe von 10-30 Jahren zur Verminderung eines Lemierre-Syndroms [1]. Die Grundlagen dazu sind aber mangelhaft: Zwar konnten beispiels- weise Centor et al. bei Studenten bei Vorliegen einer Tonsillo-Pharyngitis mittels PCR-Diagnostik den zweifachen Nachweis von Fusibakterien gegenüber GAS erbringen [4]. Ob allerdings ein ursächlicher Zusammenhang mit der Tonsillitis einerseits besteht (oder lediglich eine situativ vermehrte Kolonisation vorliegt), ist ungewiss. Andererseits ist anzuzweifeln, ob im Fall eines Nachweises von Fusibakterien die Progression zum gefährlichen LemierreSyndrom gehäuft vorkommt. Zudem stehen Studien aus, die eine Reduktion der Inzidenz des Lemierre-Syndroms unter grosszügiger antibiotischer Behandlung erbringen.

Deshalb ist es gemäss dem aktuellen Wissensstand ratsam, weiterhin einen sparsamen Umgang mit dem Einsatz von Antibiotika bei Tonsillo-Pharyngitis zu pflegen. Vielmehr müssen wir im Fall eines atypischen Verlaufs - v.a. bei Zweigipfligkeit, bei verlängerter Symptomatik (gemäss der schweizerischen Arbeitsgruppe [2] mehr als sieben Tage) oder im Fall von neuen Symptomen (z.B. Schüttelfrost oder äussere Halsschwellung) betreffend Komplikationen wie z.B. dem Lemierre-Syndrom wachsam sein.

\section{Bibliografie}

1. Gerber U, Wäckerlin A, Flury G. Eine «simple» Angina mit lebensbedrohlichen Folgen. Praxis. 2020:109:1271-1275.

2. Pelucchi C, Grigoryan L, Galeone C, et al. Guidelines der Europäischen Gesellschaft für Mikrobiologie und Infektiologie (ESCMID). Clin Microbiol Infect. 2012; Suppl. 1:1-28.

3. Hofmann Y, Bergera H, Wingeier B, et al. Behandlung der Streptokokken-Angina. Swiss Med Forum. 2019;19(2930):481488

4. Centor RM, Atkinson TP, Ratliff AE, et al. The clinical presentation of Fusobacterium-positive and streptococcal-positive pharyngitis in a university health clinic: a cross-sectional study. Ann Intern Med. 2015;162:241-247.

\section{Dr. med. Philippe Rafeiner}

FMH Innere Medizin und Infektiologie

Leitender Arzt, Stv. Chefarzt

Innere Medizin und Diagnostik

Spital Zofingen AG

Mühlethalstrasse 27

4800 Zofingen

philippe.rafeiner@spitalzofingen.ch 